\title{
A CHARACTERIZATION OF THE KERNEL OF THE POINCARÉ SERIES OPERATOR
}

\author{
MAKOTO MASUMOTO \\ Dedicated to Professor Tadashi Kuroda on his 60th birthday
}

\begin{abstract}
Let $\Gamma$ be a finitely generated Fuchsian group of the first kind acting on the unit disk $\Delta$. The kernel of the Poincaré series operator of the Hardy space $H^{p}, 1<p<\infty$, onto the Bers space $A_{q}(\Gamma)$ of integrable holomorphic automorphic forms of weight $-2 q, q \in \mathbf{Z}, q \geq 2$, on $\Delta$ for $\Gamma$ is characterized in terms of Eichler integrals of order $1-q$ on $\Delta$ for $\Gamma$.
\end{abstract}

Introduction. In $1882 \mathrm{H}$. Poincaré raised the problem, called Poincaré's vanishing problem, of finding explicit conditions that a function belongs to the kernel of the Poincaré theta series operator. In [14] G. M. Ljan proved that a certain set is dense in the kernel of the Poincaré series operator $A_{q} \rightarrow A_{q}(\Gamma)$ (for the notations, see $\S 1$ below), and improved this result in [15]. T. A. Metzger [18] obtained another dense set in the kernel (see also Kra [12]). Their results gave no algorithm whether or not a specific function belongs to the kernel, however. D. A. Hejhal first succeeded in giving a finite algorithm that determines whether or not certain rational functions are in the kernel of the Poincaré series operator (see [10] and the references given there). I. Kra [12] used the Eichler cohomology theory to consider the same problem, and he also obtained an algebraic decision procedure for the solution.

In this paper we consider a finitely generated Fuchsian group of the first kind acting on the unit disk, and give a necessary and sufficient condition that a function in the Hardy space $H^{p}, 1<p<\infty$, belongs to the kernel. The condition is stated in terms of Eichler integrals. Our theorem leads us to a result (cf. Proposition 1) similar to Kra's theorem [12, Theorem 2], which is a fundamental tool in his solution. Our approach is, however, quite different from his.

The author would like to express his sincere thanks to Professor F-Y. Maeda who gave him a great deal of advice. He also thanks Professor M. Shiba for valuable suggestions and kind guidance.

1. Main theorem. Let $\Gamma$ be a Fuchsian group keeping the unit disk $\Delta$ invariant. Fix an integer $q \geq 2$. If $D$ is a $\Gamma$-invariant open subset of the set $\Omega$ of discontinuity of $\Gamma$ and has at least three boundary points in the Riemann sphere $\hat{\mathbf{C}}=\mathbf{C} \cup\{\infty\}$, then we denote by $A_{q}(D, \Gamma)$ the complex Banach space of integrable holomorphic automorphic forms of weight $-2 q$ on $D$ for $\Gamma$; that is, a holomorphic function $\varphi$ on $D$ is, by definition, in $A_{q}(D, \Gamma)$ if

$$
\varphi(\gamma(z)) \gamma^{\prime}(z)^{q}=\varphi(z), \quad \gamma \in \Gamma, z \in D,
$$

Received by the editors November 18, 1985.

1980 Mathematics Subject Classification. Primary 30F35.

Key words and phrases. Poincaré series, automorphic form, Eichler integral.

(C) 1987 American Mathematical Society $0002-9947 / 87 \$ 1.00+\$ .25$ per page 
and

$$
\|\varphi\|_{D, \Gamma}=\iint_{D / \Gamma} \lambda_{D}(z)^{2-q}|\varphi(z)||d z \wedge d \bar{z}|<+\infty,
$$

where $\lambda_{D}(z)|d z|$ is the Poincaré metric on $D$ (with constant negative curvature -1). For simplicity we set $A_{q}(\Gamma)=A_{q}(\Delta, \Gamma),\|\varphi\|_{\Gamma}=\|\varphi\|_{\Delta, \Gamma}$ and $\lambda=\lambda_{\Delta}$. If $\Gamma=\{\mathrm{id}\}$, we abbreviate $A_{q}(\Gamma)$ and $\|\varphi\|_{\Gamma}$ to $A_{q}$ and $\|\varphi\|$, respectively.

A meromorphic function $F$ on $D$ is said to be an Eichler integral of order $1-q$ on $D$ for $\Gamma$ if $(F \circ \gamma) \cdot\left(\gamma^{\prime}\right)^{1-q}-F$ is the restriction of a polynomial of degree at most $2 q-2$ for each $\gamma \in \Gamma$. In this case it follows from Bol's formulae [5] (see also Kra [11, Lemma 4.1 in Chapter V]) that $\varphi=F^{(2 q-1)}$ is an automorphic form of weight $-2 q$ on $D$ for $\Gamma$; that is, $\varphi$ satisfies equation (1). Conversely, if $F$ is meromorphic on $D$ and $F^{(2 q-1)}$ is an automorphic form of weight $-2 q$, then $F$ is an Eichler integral of order $1-q$.

For a function $f$ defined on $\Delta$ we say that the Poincaré series $\Theta_{q} f$ exists if the series

$$
\Theta_{q} f(\varsigma)=\sum_{\gamma \in \Gamma} f(\gamma(\zeta)) \gamma^{\prime}(\zeta)^{q}
$$

converges absolutely and uniformly on compact subsets of $\Delta$. In this case $\Theta_{q} f$ is an automorphic form of weight $-2 q$. It is well known that $\Theta_{q} f$ exists for $f \in A_{q}$ and in fact $\left\|\Theta_{q} f\right\|_{\Gamma} \leq\|f\|$. Bers $\left[\mathbf{4}\right.$, Theorem 2] showed that $\Theta_{q}: A_{q} \rightarrow A_{q}(\Gamma)$ is surjective.

Let $0<p \leq \infty$. The Hardy space $H^{p}$ consists of all holomorphic functions $f$ on $\Delta$ that satisfy

$$
\begin{aligned}
\|f\|_{p} & =\sup _{0<r<1}\left\{\frac{1}{2 \pi} \int_{0}^{2 \pi}\left|f\left(r e^{i \theta}\right)\right|^{p} d \theta\right\}^{1 / p}<+\infty \text { if } 0<p<\infty, \\
\|f\|_{\infty} & =\sup _{z \in \Delta}|f(z)|<+\infty \quad \text { if } p=\infty
\end{aligned}
$$

If $1 \leq p \leq \infty$, then $H^{p}$ is a complex Banach space with norm $\|\cdot\|_{p}$. If $0<p<1$, then $H^{p}$ is a complete metric space with distance $\|f-g\|_{p}^{p}$. Every $f \in H^{p}$ admits a radial limit $\hat{f}\left(e^{i \theta}\right)=\lim _{r \rightarrow 1} f\left(r e^{i \theta}\right)$ for almost every $e^{i \theta} \in \partial \Delta$, and

$$
\begin{aligned}
\|f\|_{p} & =\left\{\frac{1}{2 \pi} \int_{0}^{2 \pi}\left|\hat{f}\left(e^{i \theta}\right)\right|^{p} d \theta\right\}^{1 / p} \quad \text { if } 0<p<\infty, \\
\|f\|_{\infty} & =\underset{\partial \Delta}{\operatorname{ess} \sup }\left|\hat{f}\left(e^{i \theta}\right)\right|, \text { if } p=\infty .
\end{aligned}
$$

Let $1 \leq p \leq \infty$ and $1 / p+1 / p^{\prime}=1$. For $f \in H^{p}$ and $g \in H^{p^{\prime}}$, we set

$$
\langle f, g\rangle=\frac{1}{2 \pi} \int_{0}^{2 \pi} \hat{f}\left(e^{i \theta}\right) \overline{\hat{g}\left(e^{i \theta}\right)} d \theta .
$$

If $1<p<\infty$, then every element $\phi$ of the conjugate space $\left(H^{p}\right)^{*}$ of $H^{p}$ is representable in the form $\phi(f)=\langle f, g\rangle$ by a unique $g \in H^{p^{\prime}}$, and the mapping $\phi \mapsto g$ is an anti-linear isomorphism of $\left(H^{p}\right)^{*}$ onto $H^{p^{\prime}}$ (cf. Duren [7, Theorem 7.3]). Thus we may identify $\left(H^{p}\right)^{*}$ with $H^{p^{\prime}}$.

Now, we are ready to state our main result. 
THEOREM. Let $\Gamma$ be a finitely generated Fuchsian group of the first kind acting on the unit disk $\Delta$. Let $1<p<\infty$ and $1 / p+1 / p^{\prime}=1$. Denote by $E_{q}^{p^{\prime}}(\Gamma)$ the set of all $g \in H^{p^{\prime}}$ such that the function $z^{2 q-1} g(z)$ is an Eichler integral of order $1-q$ on $\Delta$ for $\Gamma$. Then, the kernel $N_{q}^{p}(\Gamma)$ of the Poincaré series operator $\Theta_{q}: H^{p} \rightarrow A_{q}(\Gamma)$ is precisely the annihilator $E_{q}^{p^{\prime}}(\Gamma)^{\perp}$ of $E_{q}^{p^{\prime}}(\Gamma)$. In other words, for $f \in H^{p}$, the function $\Theta_{q} f$ vanishes identically if and only if $\langle f, g\rangle=0$ for all $g \in E_{q}^{p^{\prime}}(\Gamma)$.

2. Proof of the theorem. If $f \in H^{1}$, then $\Theta_{q} f$ exists. In fact the next lemma holds.

LEMMA 1. Let $s=1 / q$ and $f \in H^{s}$. Then $\Theta_{q} f$ exists and belongs to $A_{q}(\Gamma)$. Furthermore, the linear operator $H^{s} \ni f \mapsto \Theta_{q} f \in A_{q}(\Gamma)$ is continuous.

Proof. By Duren-Shields [8, Theorem 2], there is a positive constant $C$ such that

$$
\begin{aligned}
& \iint_{\Delta} \lambda(z)^{2-q} u(z, z, \ldots, z)^{2}|d z \wedge d \bar{z}| \\
& \quad \leq C \lim _{r \rightarrow 1} \frac{1}{(2 \pi)^{q}} \int_{0}^{2 \pi} \cdots \int_{0}^{2 \pi} u\left(r e^{i \theta_{1}}, \ldots, r e^{i \theta_{q}}\right)^{2} d \theta_{1} \cdots d \theta_{q}
\end{aligned}
$$

for all nonnegative $q$-subharmonic functions $u$ in the polydisk $\Delta^{q}$. (A function $u\left(z_{1}, \ldots, z_{q}\right)$ upper semicontinuous in a domain of $\mathbf{C}^{q}$ is said to be $q$-subharmonic if $u$ is subharmonic in each variable separately.) If $f \in H^{s}$, then, letting $u\left(z_{1}, \ldots, z_{q}\right)$ $=\Pi_{j=1}^{q}\left|f\left(z_{j}\right)\right|^{s / 2}$, we have $\|f\| \leq C\|f\|_{s}$. Thus $H^{s} \subset A_{q}$ and the inclusion map is continuous. Since the operator $A_{q} \ni f \mapsto \Theta_{q} f \in A_{q}(\Gamma)$ is continuous, the lemma is proved.

REMARK. Lemma 1 also follows from a theorem of Hardy-Littlewood (cf. Duren [7, Theorem 5.11]; see also Metzger-Sheingorn [19]). By a recent result of Mateljević and Pavlović (cf. [17]), we have $\|f\| \leq\left\{2^{3-q} \pi /(q-1)\right\}\|f\|_{s}$ and hence $\left\|\Theta_{q} f\right\|_{\Gamma} \leq$ $\left\{2^{3-q} \pi /(q-1)\right\}\|f\|_{s}$ for $f \in H^{s}$.

Let $A$ denote the class of functions holomorphic in $\Delta$ and continuous in $\bar{\Delta}$. Let $\Lambda_{*}$ be the set of all $f \in A$ such that

$$
\sup _{\theta \in \mathbf{R}, h>0} \frac{1}{h}\left|\hat{f}\left(e^{i(\theta+h)}\right)-2 \hat{f}\left(e^{i \theta}\right)+\hat{f}\left(e^{i(\theta-h)}\right)\right|<+\infty .
$$

LEMMA 2. Let $\psi_{n}(z)=z^{n}, n \in \mathbf{Z}$. Set $K_{\zeta}(z)=\sum_{n=0}^{\infty} \overline{\Theta_{q} \psi_{n}(\zeta)} z^{n}, z \in \Delta$, $\varsigma \in \Delta$. Then $K_{\zeta} \in A, K_{\zeta}^{(q-2)} \in \Lambda_{*}$, and

$$
\Theta_{q} f(\varsigma)=\lim _{r \rightarrow 1} \frac{1}{2 \pi} \int_{0}^{2 \pi} f\left(r e^{i \theta}\right) \overline{\hat{K}_{\zeta}\left(e^{i \theta}\right)} d \theta
$$

for all $f \in H^{1 / q}$. In particular, if $f \in H^{1}$, then $\Theta_{q} f(\zeta)=\left\langle f, K_{\zeta}\right\rangle, \zeta \in \Delta$.

PROOF. Lemma 1 implies that the linear functional $H^{1 / q} \ni f \mapsto \Theta_{q} f(\varsigma) \in \mathbf{C}$ is continuous for each fixed $\zeta \in \Delta$. Thus there is a unique $K_{\zeta} \in A$ such that $K_{\zeta}^{(q-2)} \in \Lambda_{*}$ and $K_{\zeta}$ satisfies (2) for all $f \in H^{1 / q}$ (cf. Duren [7, Theorem 7.5]). It is clear that

$$
K_{\zeta}(z)=\sum_{n=0}^{\infty} \overline{\Theta_{q} \psi_{n}(\varsigma)} z^{n}, \quad z \in \Delta .
$$

This completes the proof. 
Next we shall show that $K_{\zeta} \in E_{q}^{p^{\prime}}(\Gamma)$ for $\varsigma \in \Delta$, or the functions $z \mapsto z^{2 q-1} K_{\zeta}(z)$ are Eichler integrals of order $1-q$. To this end, we first state the following lemma.

Lemma 3. Let $\Delta^{*}=\hat{\mathbf{C}}-\bar{\Delta}$. A meromorphic function $F(z), z \in \Delta$, is an Eichler integral of of order $1-q$ on $\Delta$ for $\Gamma$ if and only if $z^{2 q-2} \overline{F(1 / \bar{z})}, z \in \Delta^{*}$, is an Eichler integral of order $1-q$ on $\Delta^{*}$ for $\Gamma$.

This lemma is easily verified by a direct computation.

LEMMA 4. $K_{\zeta} \in E_{q}^{p^{\prime}}(\Gamma)$ for each $\zeta \in \Delta$.

ProOF. Set

$$
L_{\zeta}(z)=\frac{1}{z} \overline{K_{\zeta}\left(\frac{1}{\bar{z}}\right)}=\frac{1}{z} \sum_{n=0}^{\infty}\left(\sum_{\gamma \in \Gamma} \gamma(\varsigma)^{n} \gamma^{\prime}(\varsigma)^{q}\right) \frac{1}{z^{n}}, \quad z \in \Delta^{*}, \zeta \in \Delta .
$$

If it is shown that we may reverse the order of summation, we will have

$$
L_{\zeta}(z)=\frac{1}{z} \sum_{\gamma \in \Gamma} \gamma^{\prime}(\varsigma)^{q}\left(\sum_{n=0}^{\infty} \frac{\gamma(\varsigma)^{n}}{z^{n}}\right)=\sum_{\gamma \in \Gamma} \frac{\gamma^{\prime}(\varsigma)^{q}}{z-\gamma(\zeta)} .
$$

But for each fixed $\zeta \in \Delta$ the last series converges absolutely and uniformly on compact subsets of $\Delta^{*}$. Thus the Weierstrass theorem on double series guarantees the interchange of the order of summation, and we obtain (4).

Now, by Ahlfors $[\mathbf{2}, \S 6.2]$ (cf. Kra [11, Lemma 7.2 in Chapter V]), the last series of (4), as a function of $z$, is an Eichler integral of order $1-q$ on $\Omega$ for $\Gamma$. This together with Lemma 3 yields the lemma.

We have not yet used the assumption that $\Gamma$ is a finitely generated Fuchsian group of the first kind. In the next lemma we need this assumption.

LEMMA 5. If $\Gamma$ is finitely generated and of the first kind, then $E_{q}^{p^{\prime}}(\Gamma)$ is spanned by $K_{\zeta}, \zeta \in \Delta$.

ProOF. The hypothesis implies that $A_{q}(\Gamma)$ is of finite dimension, and that every element of $A_{q}(\Gamma)$ is of the form $\Theta_{q} v$ with $v$ a polynomial (see, for example, Bers [3, Theorem 2]). Hence $\Theta_{q}: H^{p} \rightarrow A_{q}(\Gamma)$ is surjective, and $A_{q}(\Gamma)$ is isomorphic to $H^{p} / N_{q}^{p}(\Gamma)$, which is isomorphic to the annihilator $N_{q}^{p}(\Gamma)^{\perp}\left(\subset H^{p^{\prime}}\right)$. On the other hand, if $g \in E_{q}^{p^{\prime}}(\Gamma)$, then $(d / d z)^{2 q-1}\left\{z^{2 q-1} g(z)\right\} \in A_{q}(\Gamma)$, and this defines an isomorphism of $E_{q}^{p^{\prime}}(\Gamma)$ onto $A_{q}(\Gamma)$ (cf. [16, Theorem 3]). Therefore, we have $\operatorname{dim} N_{q}^{p}(\Gamma)^{\perp}=\operatorname{dim} E_{q}^{p^{\prime}}(\Gamma)=\operatorname{dim} A_{q}(\Gamma)<\infty$. Since $N_{q}^{p}(\Gamma)^{\perp}$ is the smallest closed subspace of $H^{p^{\prime}}$ that contains $K_{\zeta}, \varsigma \in \Delta$, we have the lemma.

In the next section, as an application of our theorem, we will prove a classical result, which implies that every element of $A_{q}(\Gamma)$ is of the form $\Theta_{q} v$ with $v$ a polynomial (see Proposition 2). This fact was used in the above proof. Therefore, it may be of some significance to give an alternative proof of Lemma 5 which does not use this fact.

Alternative PROOF OF LEMma 5. It follows from the assumption that the Riemann surface $\Delta / \Gamma$ is obtained from a closed Riemann surface $S$ by removing at most finitely many points. Since $d=\operatorname{dim} A_{q}(\Gamma)=\operatorname{dim} E_{q}^{p^{\prime}}(\Gamma)<\infty$, we have only to find $d$ linearly independent elements in $\left\{K_{\varsigma} \mid \varsigma \in \Delta\right\}$. 
Choose $d$ points $\zeta_{1}, \ldots, \zeta_{d} \in \Delta$ and $d$ forms $\varphi_{1}, \ldots, \varphi_{d} \in A_{q}(\Gamma)$ such that (i) none of $\varsigma_{j}, j=1, \ldots, d$, are elliptic fixed points, and (ii) $\varphi_{j}\left(\zeta_{k}\right)=\delta_{j k}, j, k=$ $1, \ldots, d$, where $\delta_{j k}$ is the Kronecker delta. We claim that $K_{\zeta 1}, \ldots, K_{{ }_{\zeta d}}$ are linearly independent, or equivalently, $L_{\zeta_{1}}, \ldots, L_{{ }^{\prime}}$, as functions defined on $\Delta^{*}$, are linearly independent. Assume that

$$
L(z)=\sum_{k=1}^{d} c_{k} L_{\varsigma k}(z) \equiv 0, \quad z \in \Delta^{*},
$$

for some constants $c_{1}, \ldots, c_{d}$. Recall that each $L_{\zeta}$ is an Eichler integral on $\Omega$ (see the proof of Lemma 4). Thus $L$ is also an Eichler integral on $\Omega$, whose singularities are simple poles at $\gamma\left(\zeta_{j}\right), \gamma \in \Gamma, j=1, \ldots, d$, and whose period is zero, that is, $(L \circ \gamma) \cdot\left(\gamma^{\prime}\right)^{1-q}=L$ for all $\gamma \in \Gamma$. Therefore, for each $j=1, \ldots, d$, we see that $L \varphi_{j}$ induces an Abelian differential on $S$ whose only singularity is a simple pole at the projection of $\zeta_{j}$ with residue $c_{j}$. Since $S$ is closed, we have $c_{j}=0, j=1, \ldots, d$. This completes the proof.

We can now prove the theorem easily. It follows from Lemmas 2 and 5 that for $f \in H^{p}$

$$
f \in N_{q}^{p}(\Gamma) \Leftrightarrow\left\langle f, K_{\zeta}\right\rangle=0 \text { for all } \zeta \in \Delta \Leftrightarrow f \in E_{q}^{p^{\prime}}(\Gamma)^{\perp} .
$$

This proves the theorem.

3. Consequences. In the rest of this paper we give some consequences of the theorem and the lemmas. In this section we consider finitely generated Fuchsian groups of the first kind.

If $f$ is meromorphic on $\Delta^{*}$ and has a radial limit at $e^{i \theta} \in \partial \Delta^{*}$, we denote this limit by $\hat{f}\left(e^{i \theta}\right)$.

PROPOSITION 1. Let $\Gamma$ be a finitely generated Fuchsian group of the first kind acting on the unit disk $\Delta$. Denote by $E_{1-q}\left(\Delta^{*}, \Gamma\right)$ the space of Eichler integrals of order $1-q$ on $\Delta^{*}$ for $\Gamma$ that are bounded in $\Delta^{*}$ and vanish at $\infty$. Let $a_{1}, \ldots, a_{n}, b_{1}, \ldots, b_{m}$ be distinct points in $\mathbf{C}$ such that $\left|a_{j}\right|>1, j=1, \ldots, n$, and $\left|b_{k}\right|=1, k=1, \ldots, m$. Set

$$
\begin{aligned}
r(z)= & \sum_{\nu=0}^{l_{0}} s_{\nu}(\nu+1) ! z^{\nu}+\sum_{j=1}^{n} \sum_{\nu=0}^{l_{j}} t_{j \nu} \nu !\left(z-a_{j}\right)^{-\nu-1} \\
& +\sum_{k=1}^{m} \sum_{\nu=0}^{q-2} u_{k \nu} \nu !\left(z-b_{k}\right)^{-\nu-1}
\end{aligned}
$$

where $l_{j}$ are nonnegative integers and $s_{\nu}, t_{j \nu}$ and $u_{k \nu}$ are complex numbers. Then, $\Theta_{q} r$ vanishes identically on $\Delta$ if and only if

$$
\sum_{\nu=0}^{l_{0}} s_{\nu}\left(\frac{d}{d z}\right)^{\nu+1}\left(F \circ \psi_{-1}\right)(0)-\sum_{j=1}^{n} \sum_{\nu=0}^{l_{j}} t_{j \nu} F^{(\nu)}\left(a_{j}\right)-\sum_{k=1}^{m} \sum_{\nu=0}^{q-2} u_{k \nu} \widehat{F^{(\nu)}}\left(b_{k}\right)=0
$$

for all $F \in E_{1-q}\left(\Delta^{*}, \Gamma\right)$.

Proof. Let $L_{\zeta}(z)=\overline{K_{\zeta}(1 / \bar{z})} / z, z \in \Delta^{*}$ (see (3)). Lemma 2 implies that $L_{\zeta}^{(\nu)}$, $\nu=0,1, \ldots, q-2$, are extended continuously to $\overline{\Delta^{*}}$. 
Set $r_{a, \nu}(z)=\nu !(z-a)^{-\nu-1}$, where $a \in \mathbf{C}$ and $\nu$ is a nonnegative integer. If $|a|>1$, then for $\zeta \in \Delta$ we have

$$
\Theta_{q} r_{a, \nu}(\zeta)=\left\langle r_{a, \nu}, K_{\zeta}\right\rangle=\frac{1}{2 \pi i} \int_{|z|=1} r_{a, \nu}(z) \hat{L}_{\zeta}(z) d z=-L_{\zeta}^{(\nu)}(a) .
$$

If $|b|=1$ and $0 \leq \nu \leq q-2$, then $r_{b, \nu} \in H^{1 / q}$ and $r_{R b, \nu} \rightarrow r_{b, \nu}$ in $H^{1 / q}$ as $R \downarrow 1$. Thus, by Lemma 1 ,

$$
\Theta_{q} r_{b, \nu}(\varsigma)=\lim _{R \downarrow 1} \Theta_{q} r_{R b, \nu}(\varsigma)=-\lim _{R \downarrow 1} L_{\zeta}^{(\nu)}(R b)=-\widehat{L_{\zeta}^{(\nu)}}(b) .
$$

Finally, for $\nu=0,1,2, \ldots$, we see that

$$
\Theta_{q} \psi_{\nu}(\varsigma)=\frac{1}{2 \pi i} \int_{|z|=1} z^{\nu} \hat{L}_{\zeta}(z) d z=\frac{1}{(\nu+1) !}\left(\frac{d}{d z}\right)^{\nu+1}\left(L_{\zeta} \circ \psi_{-1}\right)(0) .
$$

Since $L_{\varsigma}, \zeta \in \Delta$, span $E_{1-q}\left(\Delta^{*}, \Gamma\right)$ by Lemma 5 , we obtain the proposition.

REMARK. Kra obtained a similar result [12, Theorem 2]. Our approach is, however, quite different from his.

As an application of Proposition 1, we prove the next classical result (cf. Bers [3, Theorem 5]).

Proposition 2. Suppose that $\Gamma$ is a finitely generated Fuchsian group of the first kind and that $d=\operatorname{dim} A_{q}(\Gamma)>0$. Let $a \in \Delta$, and let $\mu_{1}, \ldots, \mu_{d}$ be the strictly increasing sequence of nonnegative integers such that for each $j=1, \ldots, d$ some $\varphi_{j} \in A_{q}(\Gamma)$ has a zero of order $\mu_{j}$ at a. Denote by $V$ the d-dimensional vector space of rational functions $v$ of the form

$$
v(z)= \begin{cases}\sum_{k=1}^{d} t_{k}\left(z-\frac{1}{\bar{a}}\right)^{-2 q-\mu_{k}} & \text { if } a \neq 0 \\ \sum_{k=1}^{d} t_{k} z^{\mu_{k}} & \text { if } a=0\end{cases}
$$

where $\left(t_{1}, \ldots, t_{d}\right) \in \mathbf{C}^{d}$. Then $\Theta_{q}$ defines a vector space isomorphism of $V$ onto $A_{q}(\Gamma)$.

ProOF. We consider only the case $a \neq 0$. The case $a=0$ can be treated similarly.

If $\varphi \in A_{q}(\Gamma)$, then $\Phi(z)=\overline{\varphi(1 / \bar{z})} z^{-2 q}, z \in \Delta^{*}$, is in $A_{q}\left(\Delta^{*}, \Gamma\right)$, and the correspondence $\varphi \mapsto \Phi$ is an antilinear isometry of $A_{q}(\Delta, \Gamma)$ onto $A_{q}\left(\Delta^{*}, \Gamma\right)$. Let $\Phi_{j}$ correspond to $\varphi_{j}, j=1, \ldots, d$. We may assume that

$$
\Phi_{j}(z)=(z-1 / \bar{a})^{\mu_{j}}+O\left(|z-1 / \bar{a}|^{\mu_{d}+1}\right) \quad(z \rightarrow 1 / \bar{a})
$$

for $j=1, \ldots, d$. There is a unique $F_{j} \in E_{1-q}\left(\Delta^{*}, \Gamma\right)$ such that $F_{j}^{(2 q-1)}=\Phi_{j}$ for each $j=1, \ldots, d$.

If $v(z)=\sum_{k=1}^{d} t_{k}(z-1 / \bar{a})^{-2 q-\mu_{k}}$ and $\Theta_{q} v$ vanishes identically on $\Delta$, then it follows from Proposition 1 that

$$
\begin{aligned}
0 & =\sum_{k=1}^{d} \frac{t_{k}}{\left(2 q+\mu_{k}-1\right) !} F_{j}^{\left(2 q+\mu_{k}-1\right)}\left(\frac{1}{\bar{a}}\right) \\
& =\sum_{k=1}^{d} \frac{t_{k}}{\left(2 q+\mu_{k}-1\right) !} \Phi_{j}^{\left(\mu_{k}\right)}\left(\frac{1}{\bar{a}}\right)=\frac{\mu_{j} !}{\left(2 q+\mu_{j}-1\right) !} t_{j},
\end{aligned}
$$


for $j=1, \ldots, d$. Thur $\Theta_{q}: V \rightarrow A_{q}(\Gamma)$ is injective and hence bijective.

REMARKS. (i) This proposition shows that every element of $A_{q}(\Gamma)$ is of the form $\Theta_{q} v$ with $v$ a polynomial if $\Gamma$ is finitely generated and of the first kind.

(ii) We can generalize Propositions 1 and 2 as follows. Let $\Gamma$ be a finitely generated nonelementary Kleinian group, and let $\Omega$ denote the set of discontinuity of $\Gamma$. Assume that $\Omega$ has a $\Gamma$-invariant component $D^{*}$ such that $\infty \in D^{*}$ and $D=\Omega-D^{*} \neq \varnothing$. Let $E_{1-q}\left(D^{*}, \Gamma\right)$ be the vector space of Eichler integrals of order $1-q$ on $D^{*}$ for $\Gamma$ that are bounded in the sense of Kra and vanish at $\infty$; that is, $E_{1-q}\left(D^{*}, \Gamma\right)$ consists of all Eichler integrals $F$ on $D^{*}$ with $F^{(2 q-1)} \in A_{q}\left(D^{*}, \Gamma\right)$ and $F(\infty)=0$ (cf. Kra [11, Chapter V, §4]). For each $\varsigma \in D$,

$$
L_{\varsigma}(z)=\sum_{\gamma \in \Gamma} \frac{\gamma^{\prime}(\varsigma)^{q}}{z-\gamma(\varsigma)}, \quad z \in \Omega
$$

is an Eichler integral on $\Omega$ and the restriction of $L_{\zeta}$ to $D^{*}$ belongs to $E_{1-q}\left(D^{*}, \Gamma\right)$. For $a \in D^{*}-\{\infty\}$, taking a subdomain $D_{0}^{*}$ of $D^{*}$ whose boundary consists of finitely many analytic curves and which contains $a$ and $\infty$, we have

$$
\begin{aligned}
\Theta_{q} r_{a, \nu}(\zeta) & =\sum_{\gamma \in \Gamma} r_{a, \nu}(\gamma(\zeta)) \gamma^{\prime}(\zeta)^{q} \\
& =\frac{1}{2 \pi i} \int_{\partial D_{0}^{*}} r_{a, \nu}(z) L_{\zeta}(z) d z=-L_{\zeta}^{(\nu)}(a),
\end{aligned}
$$

for each $\zeta \in \Delta$. Similarly, we have

$$
\Theta_{q} \psi_{\nu}(\zeta)=\frac{1}{(\nu+1) !}\left(\frac{d}{d z}\right)^{\nu+1}\left(L_{\zeta} \circ \psi_{-1}\right)(0)
$$

for $\nu=0,1,2, \ldots$ Assume now that $E_{1-q}\left(D^{*}, \Gamma\right)$ is spanned by $L_{\varsigma}, \varsigma \in D$. Then,

$$
\Theta_{q}\left(\sum_{\nu} s_{\nu}(\nu+1) ! \psi_{\nu}+\sum_{a, \nu} t_{a, \nu} r_{a, \nu}\right) \equiv 0 \text { on } D
$$

( $s_{\nu}$ and $t_{a, \nu}$ are constants) if and only if

$$
\sum_{\nu} s_{\nu}\left(\frac{d}{d z}\right)^{\nu+1}\left(F \circ \psi_{-1}\right)(0)-\sum_{a, \nu} t_{a, \nu} F^{(\nu)}(a)=0
$$

for all $F \in E_{1-q}\left(D^{*}, \Gamma\right)$. This is a generalization of Proposition 1. Using this, we can easily generalize Proposition 2. In particular, every element of $A_{q}(D, \Gamma)$ is of the form $\Theta_{q} v$ with $v$ a polynomial. Note that $E_{1-q}\left(D^{*}, \Gamma\right)$ is spanned by $L_{\varsigma}, \zeta \in D$, for finitely generated quasi-Fuchsian groups $\Gamma$ of the first kind. (For such $\Gamma$ the second proof of Lemma 5 remains valid with obvious modifications.)

4. Arbitrary Fuchsian groups. In this section $\Gamma$ is assumed to be an arbitrary Fuchsian group acting on $\Delta ; \Gamma$ may be infinitely generated or of the second kind.

PROPOSITION 3 .

$$
\sum_{n=0}^{\infty}\left|\Theta_{q} \psi_{n}(\varsigma)\right|^{2} \leq \lambda(\varsigma)^{2}\left(\sum_{\gamma \in \Gamma}\left|\gamma^{\prime}(\varsigma)\right|^{q-1}\right)^{2}, \quad \varsigma \in \Delta .
$$


ProOF. Using (3) and (4), we obtain

$$
\begin{aligned}
\left\|K_{\zeta}\right\|_{\infty} & =\sup _{z \in \Delta^{*}}\left|L_{\zeta}(z)\right| \leq \sup _{z \in \Delta^{*}} \sum_{\gamma \in \Gamma} \frac{\left|\gamma^{\prime}(\varsigma)\right|^{q}}{|z-\gamma(\varsigma)|} \\
& \leq 2 \sum_{\gamma \in \Gamma} \frac{\left|\gamma^{\prime}(\zeta)\right|^{q}}{1-|\gamma(\varsigma)|^{2}}=\lambda(\varsigma) \sum_{\gamma \in \Gamma}\left|\gamma^{\prime}(\zeta)\right|^{q-1} .
\end{aligned}
$$

Thus, by the Parseval identity, we have

$$
\sum_{n=0}^{\infty}\left|\Theta_{q} \psi_{n}(\zeta)\right|^{2}=\left\|K_{\zeta}\right\|_{2}^{2} \leq\left\|K_{\zeta}\right\|_{\infty}^{2} \leq \lambda(\zeta)^{2}\left(\sum_{\gamma \in \Gamma}\left|\gamma^{\prime}(\zeta)\right|^{q-1}\right)^{2}
$$

REMARKS. (i) The series on the right of (5) converges if $q \geq 3$ or $\Gamma$ is of convergence type, while the series on the left always converges. ( $\Gamma$ is said to be of convergence type if $\sum_{\gamma \in \Gamma}(1-|\gamma(0)|)<+\infty$.)

(ii) Inequality (5) remains valid if $\left\{\psi_{n}\right\}_{n=0,1,2, \ldots}$ is a complete orthonormal system for the Hilbert space $H^{2}$.

A holomorphic automorphic form $\varphi$ of weight $-2 q$ on $\Delta$ for $\Gamma$ is said to be bounded if $\sup _{z \in \Delta} \lambda(z)^{-q}|\varphi(z)|<+\infty$. We denote by $B_{q}(\Gamma)$ the complex Banach space of all such $\varphi$.

PROPOSITION 4. If $q \geq 3$ or $\Gamma$ is of convergence type, then $\Theta_{q} f \in B_{q}(\Gamma)$ for all $f \in H^{1}$.

PROOF. If $f \in H^{1}$, then it follows from (2) and (6) that

$$
\left|\Theta_{q} f(\zeta)\right| \leq\|f\|_{1}\left\|K_{\zeta}\right\|_{\infty} \leq 2^{1-q}\|f\|_{1} \lambda(\varsigma)^{q} \sum_{\gamma \in \Gamma}\left(1-|\gamma(\zeta)|^{2}\right)^{q-1} .
$$

Since the assumption implies that $\sup _{\zeta \in \Delta} \sum_{\gamma \in \Gamma}\left(1-|\gamma(\varsigma)|^{2}\right)^{q-1}<+\infty$ (Godement [9], Ahlfors [1], Rajeswara Rao [21, (5.3)]; see also Kra [11, Lemma 5.2 in Chapter III]), we have that $\Theta_{q} f$ is bounded.

REMARKS. (i) Our proof shows that $\Theta_{q}: H^{1} \rightarrow B_{q}(\Gamma)$ is continuous.

(ii) If $\Gamma$ is finitely generated, then $A_{q}(\Gamma) \subset B_{q}(\Gamma)$ (Drasin-Earle [6, Theorem 1]). The same conclusion holds for more general $\Gamma$ (cf. Lehner $[\mathbf{1 3}]$ ). Thus for such $\Gamma$ Proposition 4 is a trivial consequence of Lemma 1 . However, it should be noted that there exists a group $\Gamma$ of convergence type for which $A_{2}(\Gamma) \not \subset B_{2}(\Gamma)$ (Pommerenke [20, Theorem 1]).

For general $\Gamma$ we may define $N_{q}^{p}(\Gamma)$ and $E_{q}^{p^{\prime}}(\Gamma)$ as in the theorem. The theorem claims that $N_{q}^{p}(\Gamma)=E_{q}^{p^{\prime}}(\Gamma)^{\perp}$ if $\Gamma$ is finitely generated and of the first kind. For arbitrary $\Gamma$ we can prove the following proposition.

Proposition 5. Let $1<p<\infty$ and $1 / p+1 / p^{\prime}=1$. Denote by $\tilde{N}_{q}^{p}(\Gamma)$ the smallest closed subspace of $H^{p}$ containing $\left(\psi_{n} \circ \gamma\right) \cdot\left(\gamma^{\prime}\right)^{q}-\psi_{n}, \gamma \in \Gamma, n=0,1,2, \ldots$ Then $\tilde{N}_{q}^{p}(\Gamma)$ coincides with the annihilator $E_{q}^{p^{\prime}}(\Gamma)^{\perp}$ of $E_{q}^{p^{\prime}}(\Gamma)$.

PROOF. If $\gamma$ is a conformal automorphism of $\Delta$, then

$$
\left\langle\left(\psi_{n} \circ \gamma^{-1}\right) \cdot\left(\left(\gamma^{-1}\right)^{\prime}\right)^{q}, g\right\rangle=\left\langle\psi_{2 q-1+n},\left(\psi_{2 q-1} \circ \gamma\right) \cdot(g \circ \gamma) \cdot\left(\gamma^{\prime}\right)^{1-q}\right\rangle, \quad g \in H^{p^{\prime}},
$$


for any nonnegative integer $n$. Thus,

$\left\langle\psi_{2 q-1+n},\left(\psi_{2 q-1} \circ \gamma\right) \cdot(g \circ \gamma) \cdot\left(\gamma^{\prime}\right)^{1-q}-\psi_{2 q-1} g\right\rangle=\left\langle\left(\psi_{n} \circ \gamma^{-1}\right) \cdot\left(\left(\gamma^{-1}\right)^{\prime}\right)^{q}-\psi_{n}, g\right\rangle$, which establishes the proposition.

COROLlaRY. Let $1<p<\infty$. Suppose that $\Gamma$ is finitely generated and of the first kind. Then, the kernel of the Poincaré series opeator $\Theta_{q}: H^{p} \rightarrow A_{q}(\Gamma)$ is identical with the smallest closed subspace of $H^{p}$ containing $\left(\psi_{n} \circ \gamma\right) \cdot\left(\gamma^{\prime}\right)^{q}-\psi_{n}$, $\gamma \in \Gamma, n=0,1,2, \ldots$

REMARKS. (i) It is always true that $E_{q}^{p^{\prime}}(\Gamma)^{\perp} \subset N_{q}^{p}(\Gamma)$ and $\tilde{N}_{q}^{p}(\Gamma) \subset N_{q}^{p}(\Gamma)$. Proposition 5 implies that $E_{q}^{p^{\prime}}(\Gamma)^{\perp}=N_{q}^{p}(\Gamma)$ if and only if $\tilde{N}_{q}^{p}(\Gamma)=N_{q}^{p}(\Gamma)$. The condition $N_{q}^{p}(\Gamma)=E_{q}^{p^{\prime}}(\Gamma)^{\perp}$ means that $E_{q}^{p^{\prime}}(\Gamma)$ is the smallest closed subspace of $H^{p^{\prime}}$ containing $K_{\zeta}, \zeta \in \Delta$.

(ii) Metzger [18] proved that the kernel of $\Theta_{q}: A_{q} \rightarrow A_{q}(\Gamma)$ is the smallest closed subspace of $A_{q}$ containing $\left(\psi_{n} \circ \gamma\right) \cdot\left(\gamma^{\prime}\right)^{q}-\psi_{n}, \gamma \in \Gamma, n=0,1,2, \ldots$

\section{REFERENCES}

1. L. V. Ahlfors, Eine Bemerkung über Fuchssche Gruppen, Math. Z. 84 (1964), 244-245.

2. _ The structure of a finitely generated Kleinian group, Acta Math. 122 (1969), 1-17.

3. L. Bers, Completeness theorems for Poincaré series in one variable, Proc. Internat. Sympos. Linear Spaces (Jerusalem, 1960), Jerusalem Academic Press, Jerusalem; Pergamon, Oxford, 1961, pp. 88-100.

4. __ Automorphic forms and Poincaré series for infinitely generated Fuchsian groups, Amer. J. Math. 87 (1965), 196-214.

5. G. Bol, Invarianten linearer Differentialgleichungen, Abh. Math. Sem. Univ. Hamburg 16 (1949), 1-28.

6. D. Drasin and C. J. Earle, On the boundedness of automorphic forms, Proc. Amer. Math. Soc. 19 (1968), 1039-1042.

7. P. L. Duren, Theory of $H^{p}$ spaces, Academic Press, New York, 1970.

8. P. L. Duren and A. L. Shields, Restriction of $H^{p}$ functions to the diagonal of the polydisc, Duke Math. J. 42 (1975), 751-753.

9. R. Godement, Série de Poincaré et Spitzenformen, Séminaire Henri Cartan, 10e année (1957/1958), exposé 10.

10. D. A. Hejhal, Monodromy groups and Poincaré series, Bull. Amer. Math. Soc. 84 (1978), 339376.

11. I. Kra, Automorphic forms and Kleinian groups, Benjamin, Reading, Mass., 1972.

12. __ On the vanishing of and spanning sets for Poincaré series for cusp forms, Acta Math. 153 (1984), 47-116.

13. J. Lehner, On the boundedness of integrable automorphic forms, Illinois J. Math. 18 (1974), 575584.

14. G. M. Ljan, On the kernel of Poincaré's $\theta$-operator, Soviet Math. Dokl. 17 (1976), 1283-1285.

15. 1977, no. 12, 7-8. (Russian)

16. M. Masumoto, On periods of certain Eichler integrals for Kleinian groups, Hiroshima Math. J. 15 (1985), 645-662.

17. M. Mateljević and M. Pavlović, Some inequalities of isoperimetric type for the integral means of analytic functions, Mat. Vesnik 37 (1985), 78-80.

18. T. A. Metzger, The kernel of the Poincaré series operator, Proc. Amer. Math. Soc. 76 (1979), 289-292.

19. T. A. Metzger and M. Sheingorn, Polynomial approximation in the Bers spaces, Ann. of Math. Studies, no. 79, Princeton Univ. Press, Princeton, N. J., 1974, pp. 369-377. 
20. Ch. Pommerenke, On inclusion relations for spaces of automorphic forms, Advances in Complex Function Theory (Proc. Sem., Univ. Maryland, College Park, Md., 1973-1974), Lecture Notes in Math., vol. 505, Springer, Berlin, 1976, pp. 92-100.

21. K. V. Rasjeswara Rao, Fuchsian groups of convergence type and Poincaré series of dimension -2 , J. Math. Mech. 18 (1969), 629644.

Department of MAthematics, Hiroshima University, Hiroshima 730, Japan 